Bull. Austral. Math. Soc.

Vol. 54 (1996) [451-458]

\title{
ON THE DARBOUX PROBLEM OF NEUTRAL TYPE
}

Dariusz Bugajewsi and Miroseawa Zima

The aim of this paper is to prove uniqueness theorems for the Darboux problem of neutral type in the space $L^{\infty}$ and $L^{1}$.

\section{INTRODUCTION}

Let $I=[0, a], a>0$. Denote by $L^{\infty}\left(I^{2}\right)$ the space of Lebesgue measurable and essentially bounded functions $z: I^{2} \rightarrow \mathbb{R}$, with the norm

$$
\|z\|_{\infty}=\operatorname{ess} \sup _{I^{2}}|z(x, y)| .
$$

Furthermore, let $L^{1}\left(I^{2}\right)$ denote the space of Lebesgue measurable functions $z: I^{2} \rightarrow \mathbb{R}$ such that $\int_{I^{2}}|z(x, y)| d x d y<+\infty$, with the norm

$$
\|z\|_{1}=\int_{I^{2}}|z(x, y)| d x d y .
$$

In this paper we consider the following Darboux problem of neutral type

$$
\begin{aligned}
z_{x y} & =f\left(x, y, z(h(x, y)), z_{x y}(H(x, y))\right), \quad(x, y) \in I^{2}, \\
z(x, 0) & =0, \quad x \in I, \\
z(0, y) & =0, \quad y \in I .
\end{aligned}
$$

In Section 2 we show that under suitable assumptions on the functions $f, h$ and $H$, the problem (1) has a unique solution in the space $L^{\infty}\left(I^{2}\right)$. To prove this we apply the fixed point theorem from the paper [7]. In Section 3 we apply the classical Banach contraction principle to obtain an analogous result for the problem (1) in the space $L^{1}\left(I^{2}\right)$. Similar problems (with or without translation of arguments) have been considered for example, in the papers $[\mathbf{2}, 3,9]$ and in the monograph [1].

In what follows we shall need two propositions from the papers [7] and [8].

Received 3rd January, 1996

The authors wish to express their thanks to Dr. A. Soltysiak for his suggestions.

Copyright Clearance Centre, Inc. Serial-fee code: 0004-9729/96 \$A2.00+0.00. 
Let $(X,\|\cdot\|, \prec, m)$ be a Banach space with a binary relation $\prec$ and a mapping $m: X \rightarrow X$. Suppose that:

(i) the relation $\prec$ is transitive,

(ii) the norm $\|\cdot\|$ is monotonic, that is, if $\theta \prec w \prec v$, then $\|w\| \leqslant\|v\|$,

(iii) $\theta \prec m(w)$ and $\|m(w)\|=\|w\|$ for all $w \in X$.

Proposition 1. [7] In the Banach space considered above, let the operators $\mathcal{A}: X \rightarrow X, A: X \rightarrow X$ be given with the following properties:

(iv) $A$ is a linear bounded operator with spectral radius $r(A)$ less than 1 ,

(v) if $\theta \prec w \prec v$, then $A w \prec A v$,

(vi) $m(\mathcal{A} w-\mathcal{A} v) \prec A m(w-v)$ for all $w, v \in X$.

Then the equation $\mathcal{A} x=x$ has a unique solution in $X$.

Assume further that:

(vii) the relation $\prec$ is reflexive,

(viii) if $w \prec v$, then $w+u \prec v+u$ for $w, v, u \in X$.

Proposition 2. [8] Let $(X,\|\cdot\|, \prec)$ denote a Banach space with a binary relation $\prec$ satisfying conditions (i), (ii), (vii) and (viii). In this space, let the linear and bounded operators $A: X \rightarrow X, B: X \rightarrow X$ be given. Assume that the following conditions are satisfied:

(ix) if $\theta \prec w$, then $\theta \prec A w$ and $\theta \prec B w$,

(x) there exists an element $w_{0} \in X, \theta \prec w_{0}$ such that $r(A+B)=$ $\lim _{n \rightarrow \infty}\left\|(A+B)^{n} w_{0}\right\|^{1 / n}$ and $B A^{j} B^{k} w_{0} \prec A^{j} B^{k+1} w_{0}$ for $j=1,2, \ldots$, $k=0,1, \ldots$.

Then the inequality

$$
r(A+B) \leqslant r(A)+r(B)
$$

holds.

\section{The Darboux problem in the space $L^{\infty}\left(I^{2}\right)$.}

Let $w, v \in L^{\infty}\left(I^{2}\right)$. We shall say that $w \prec v$ if and only if $w(x, y) \leqslant v(x, y)$ almost everywhere on $I^{2}$. Moreover, let $m(w)(x, y)=|w(x, y)|$ for $(x, y) \in I^{2}$. It is clear that the conditions (i)-(iii) and (vii)-(viii) are satisfied in this case.

Assume that:

$1^{0} h: I^{2} \rightarrow I^{2}$ is a continuous function and $h(x, y) \leqslant(x, y)$ for every pair $(x, y) \in I^{2}$, where $h(x, y)=\left(h_{1}(x, y), h_{2}(x, y)\right)$ and $\left(x_{1}, y_{1}\right) \leqslant\left(x_{2}, y_{2}\right)$ means that $x_{1} \leqslant x_{2}$ and $y_{1} \leqslant y_{2}$;

$2^{0} u \subset \mathbb{R}^{2}$ is an open set such that $I^{2} \subset u$ and $H: u \rightarrow \mathbb{R}^{2}$ is a diffeomorphism "into" with the property $H\left(I^{2}\right) \subset I^{2}$, and $h(H(x, y)) \leqslant h(x, y)$ for $(x, y) \in I^{2}$; 
$3^{0}(x, y, u, v) \rightarrow f(x, y, u, v)$ is a real function defined on the product $I^{2} \times \mathbb{R}^{2}$, Lebesgue measurable with respect to $(x, y)$ for all $(u, v) \in \mathbb{R}^{2}$ and satisfying the Lipschitz condition

$$
\left|f\left(x, y, u_{1}, v_{1}\right)-f\left(x, y, u_{2}, v_{2}\right)\right| \leqslant L_{1}\left|u_{1}-u_{2}\right|+L_{2}\left|v_{1}-v_{2}\right|
$$

for $\left(x, y, u_{1}, v_{1}\right),\left(x, y, u_{2}, v_{2}\right) \in I^{2} \times \mathbb{R}^{2}$, where $L_{1}>0$ and $0<L_{2}<1$;

$4^{0}|f(x, y, 0,0)|$ is an essentially bounded function on $I^{2}$.

By a solution of the problem (1), defined on the set $I^{2}$, we understand a function $z: I^{2} \rightarrow \mathbb{R}$ such that $z(x, y)$ is an absolutely continuous (shortly: $\mathrm{AC}$ ) function with respect to $x$ and $y, z_{x}$ is an AC-function with respect to $y$ for almost all $x \in I, z_{y}$ is an AC-function with respect to $x$ for almost all $y \in I$, $z_{x y}(x, y)=f\left(x, y, z(h(x, y)), z_{x y}(H(x, y))\right)$ almost everywhere on $I^{2}, z(x, 0)=0$ for $x \in I$ and $z(0, y)=0$ for $y \in I$.

Theorem 1. Under the assumptions $1^{0}-4^{0}$ the problem (1) has a unique solution defined on $I^{2}$.

Proof: It is easy to verify that the problem (1) is equivalent to the following functional-integral equation

$$
w(x, y)=f\left(x, y, \int_{D(h(x, y))} w(t, s) d t d s, w(H(x, y))\right), \quad(x, y) \in I^{2}
$$

where $D(x, y)=\left\{(t, s) \in I^{2}: 0 \leqslant t \leqslant x, 0 \leqslant s \leqslant y\right\}$.

Indeed, let $z: I^{2} \rightarrow \mathbb{R}$ be a solution of the problem (1) and put $z_{x y}(x, y)=w(x, y)$, $(x, y) \in I^{2}$. By the definition of a solution of (1) we have

$$
\begin{aligned}
\int_{D(h(x, y))} w(t, s) d t d s & =\int_{0}^{h_{1}(x, y)} \int_{0}^{h_{2}(x, y)} z_{\xi \eta}(\xi, \eta) d \xi d \eta \\
& =\int_{0}^{h_{2}(x, y)}\left[\int_{0}^{h_{1}(x, y)} \frac{\partial}{\partial \xi}\left(\frac{\partial}{\partial \eta} z(\xi, \eta)\right) d \xi\right] d \eta \\
& =\int_{0}^{h_{2}(x, y)}\left[\frac{\partial}{\partial \eta} z\left(h_{1}(x, y), \eta\right)-\frac{\partial}{\partial \eta} z(0, \eta)\right] d \eta \\
& =\int_{0}^{h_{2}(x, y)} \frac{\partial}{\partial \eta} z\left(h_{1}(x, y), \eta\right) d \eta
\end{aligned}
$$




$$
\begin{aligned}
& =z\left(h_{1}(x, y), h_{2}(x, y)\right)-z\left(h_{1}(x, y), 0\right) \\
& =z\left(h_{1}(x, y), h_{2}(x, y)\right)=z(h(x, y)) .
\end{aligned}
$$

This means that $w: I^{2} \rightarrow \mathbb{R}$ is a solution of the equation (2). On the other hand, let $w: I^{2} \rightarrow \mathbb{R}$ be a solution of (2) in the space $L^{\infty}\left(I^{2}\right)$. Put $z(x, y)=\int_{0}^{x} \int_{0}^{y} w(\xi, \eta) d \xi d \eta$. By the Tolstov theorem [6], we have

$$
z_{x y}(x, y)=\frac{\partial^{2}}{\partial x \partial y} \int_{0}^{z} \int_{0}^{y} w(\xi, \eta) d \xi d \eta=w(x, y) \quad \text { for almost all } \quad(x, y) \in I^{2}
$$

Thus $z_{x y}(H(x, y))=w(H(x, y))$ for almost all $(x, y) \in I^{2}$ and in consequence $z: I^{2} \rightarrow$ $\mathbb{R}$ is a solution of (1).

Consider the following operator:

$$
F(w)(x, y)=f\left(x, y, \int_{D(h(x, y))} w(t, s) d t d s, w(H(x, y))\right)
$$

where $w \in L^{\infty}\left(I^{2}\right),(x, y) \in I^{2}$.

Since the function $(x, y) \rightarrow \int_{D(h(x, y))} w(t, s) d t d s$ is continuous on $I^{2}$ and the function $(x, y) \rightarrow w(H(x, y))$ is Lebesgue integrable on $I^{2}$, the function $(x, y) \rightarrow$ $f\left(x, y, \int_{D(h(x, y))} w(t, s) d t d s, w(H(x, y))\right)$ is Lebesgue measurable on $I^{2}$. Moreover, in view of $3^{0}$ we have

$$
\begin{aligned}
|F(w)(x, y)| & \leqslant\left. L_{1}\right|_{D(h(x, y))} w(t, s) d t d s\left|+L_{2}\right| w(H(x, y))|+| f(x, y, 0,0) \mid \\
& \leqslant L_{1} a^{2}\|w\|_{\infty}+L_{2}\|w\|_{\infty}+|f(x, y, 0,0)|
\end{aligned}
$$

for $w \in L^{\infty}\left(I^{2}\right)$ and $(x, y) \in I^{2}$. Hence, by the above inequality and $4^{0}, F\left(L^{\infty}\left(I^{2}\right)\right) \subset$ $L^{\infty}\left(I^{2}\right)$. Again in view of $3^{0}$, for $w, v \in L^{\infty}\left(I^{2}\right),(x, y) \in I^{2}$ we get

$$
\begin{aligned}
& |F(w)(x, y)-F(v)(x, y)| \\
& =\mid f\left(x, y, \int_{D(h(x, y))} w(t, s) d t d s, w(H(x, y))\right) \\
& -f\left(x, y, \int_{D(h(x, y))} v(t, s) d t d s, v(H(x, y))\right) \mid \\
& \quad \leqslant L_{1} \int_{D(h(x, y))}|w(t, s)-v(t, s)| d t d s+L_{2}|w(H(x, y))-v(H(x, y))| .
\end{aligned}
$$


Thus

$$
|F(w)(x, y)-F(v)(x, y)| \leqslant\left(A_{1}+A_{2}\right)(|w-v|)(x, y)
$$

where $A_{1}(u)(x, y)=L_{1} \int_{D(h(x, y))} u(t, s) d t d s, A_{2}(u)(x, y)=L_{2} u(H(x, y)), u \in L^{\infty}\left(I^{2}\right)$.

We shall show now that the operators $A_{1}+A_{2}$ and $F$ satisfy the assumptions of Proposition 1. Obviously, the operator $A_{1}+A_{2}$ is linear and $\left(A_{1}+A_{2}\right)\left(L^{\infty}\left(I^{2}\right)\right) \subset$ $L^{\infty}\left(I^{2}\right)$. Furthermore

$$
\begin{aligned}
\left\|\left(A_{1}+A_{2}\right) w\right\|_{\infty} & =\operatorname{ess} \sup _{I^{2}}\left|L_{1} \int_{D(h(x, y))} w(t, s) d t d s+L_{2} w(H(x, y))\right| \\
& \leqslant L_{1} \text { ess } \sup _{I^{2}} \int_{D(h(x, y))}|w(t, s)| d t d s+L_{2} \text { ess } \sup _{I^{2}}|w(H(x, y))| \\
& \leqslant L_{1} a^{2}\|w\|_{\infty}+L_{2}\|w\|_{\infty},
\end{aligned}
$$

which means that $A_{1}+A_{2}$ is a bounded operator. Moreover, $A_{1}+A_{2}$ is an increasing operator. Indeed, if $w, v \in L^{\infty}\left(I^{2}\right)$ and $\theta \prec w \prec v$, then for almost all $(x, y) \in I^{2}$ we have

$$
\begin{aligned}
\left(A_{1}+A_{2}\right)(w)(x, y) & =L_{1} \int_{D(h(x, y))} w(t, s) d t d s+L_{2} w(H(x, y)) \\
& \leqslant L_{1} \int_{D(h(x, y))} v(t, s) d t d s+L_{2} v(H(x, y))=\left(A_{1}+A_{2}\right)(v)(x, y) .
\end{aligned}
$$

Notice that in view of (3) the condition (vi) of Proposition 1 is satisfied. It remains to prove that $r\left(A_{1}+A_{2}\right)<1$. First we shall show that the operators $A_{1}, A_{2}$ and $A_{1}+A_{2}$ satisfy the assumptions of Proposition 2. For $\theta \prec w$ we have $\theta \prec A_{1} w$ and $\theta \prec A_{2} w$. Let $K$ denote a cone of nonnegative functions in $L^{\infty}\left(I^{2}\right)$, that is, $K=\left\{w \in L^{\infty}\left(I^{2}\right): w(x, y) \geqslant 0\right.$ almost everywhere on $\left.I^{2}\right\}$ and let $w_{0}(x, y)=1$ almost everywhere on $I^{2}$. It is easy to verify that the cone $K$ is normal and $w_{0} \in$ intK . Hence $r\left(A_{1}+A_{2}\right)=\lim _{n \rightarrow \infty}\left\|\left(A_{1}+A_{2}\right) w_{0}\right\|_{\infty}^{1 / n}$ (for example, see $[4,5]$ ). For $j=1,2, \ldots$, $k=0,1, \ldots$, we have

$$
\begin{aligned}
& A_{2} A_{1}^{j} A_{2}^{k}\left(w_{0}\right)(x, y) \\
& =L_{1}^{j} L_{2}^{k+1} \int_{D(h(H(x, y)))} \int_{D\left(h\left(x_{1}, y_{1}\right)\right)} \cdots \int_{D\left(h\left(x_{j-1}, y_{j-1}\right)\right)} 1 d x_{j} d y_{j} \ldots d x_{1} d y_{1}
\end{aligned}
$$


and

$$
\begin{aligned}
& A_{1}^{j} A_{2}^{k+1}\left(w_{0}\right)(x, y) \\
& =L_{1}^{j} L_{2}^{k+1} \int_{D(h(x, y))} \int_{D\left(h\left(x_{1}, y_{1}\right)\right)} \cdots \int_{D\left(h\left(x_{j-1}, y_{j-1}\right)\right)} 1 d x_{j} d y_{j} \ldots d x_{1} d y_{1} .
\end{aligned}
$$

Hence, in view of $2^{0}$,

$$
A_{2} A_{1}^{j} A_{2}^{k} w_{0} \prec A_{1}^{j} A_{2}^{k+1} w_{0}
$$

for $j=1,2, \ldots, k=0,1, \ldots$ Therefore, by Proposition 2 , the inequality $r\left(A_{1}+A_{2}\right) \leqslant$ $r\left(A_{1}\right)+r\left(A_{2}\right)$ holds. Finally, an easy computation shows that $\left\|A_{1}^{n} w_{0}\right\|_{\infty} \leqslant$ $\left(L_{1}^{n} a^{2 n}\right) /(n !)^{2}$ while $\left\|A_{2}^{n} w_{0}\right\|_{\infty}=L_{2}^{n}$. Thus $r\left(A_{1}\right)=0$ and $r\left(A_{2}\right)=L_{2}$. Since $L_{2}<1$, this gives $r\left(A_{1}+A_{2}\right)<1$. It follows from Proposition 1 that the equation (2) has exactly one solution in $L^{\infty}\left(I^{2}\right)$. This completes the proof of Theorem 1 .

\section{The Darboux problem in the space $L^{1}\left(I^{2}\right)$}

Assume now that

$5^{0} \quad h: I^{2} \rightarrow I^{2}$ is a continuous function;

$6^{0} U, V \subset \mathbb{R}^{2}$ are any open sets such that $I^{2} \subset U, I^{2} \subset V$ and $H: U \rightarrow V$ is a diffeomorphism with the property $H\left(I^{2}\right)=I^{2}$;

$7^{0}(x, y, u, v) \rightarrow f(x, y, u, v)$ is a real function defined on the product $I^{2} \times \mathbb{R}^{2}$ which is Lebesgue measurable in $(x, y)$ for every $(u, v) \in \mathbb{R}^{2}$ and satisfies the Lipschitz condition

$$
\left|f\left(x, y, u_{1}, v_{1}\right)-f\left(x, y, u_{2}, v_{2}\right)\right| \leqslant L_{1}\left|u_{1}-u_{2}\right|+L_{2}\left|v_{1}-v_{2}\right|
$$

for $\left(x, y, u_{1}, v_{1}\right),\left(x, y, u_{2}, v_{2}\right) \in I^{2} \times \mathbb{R}^{2}$, where $L_{1}, L_{2}>0, L_{1} a^{2}+L_{2} M<1, M=$ $\left(\min _{(x, y) \in I^{2}}\left|H^{\prime}(x, y)\right|\right)^{-1}$ and $\left|H^{\prime}(x, y)\right|$ denotes the absolute value of the Jacobian of the mapping $H$;

$8^{0}$ there exists a function $m_{0}: I^{2} \rightarrow \mathbb{R}_{+}$which is integrable in the Lebesgue sense and such that

$$
|f(x, y, 0,0)| \leqslant m_{0}(x, y) \quad \text { for } \quad(x, y) \in I^{2}
$$

In the situation described above, we define a solution of (1) on $I^{2}$ analogously as in the previous section.

Now we can prove the following 
THEOREM 2. Under the above assumptions the problem (1) has a unique solution defined on $I^{2}$.

ProOf: The same arguments as in the proof of Theorem 1 show that (1) and (2) are equivalent. Define the operator

$$
F(w)(x, y)=f\left(x, y, \int_{D(h(x, y))} w(t, s) d t d s, w(H(x, y))\right)
$$

where $w \in L^{1}\left(I^{2}\right),(x, y) \in I^{2}$.

Since

$$
|F(w)(x, y)| \leqslant L_{1}\left|\int_{D(h(x, y))} w(t, s) d t d s\right|+L_{2}|w(H(x, y))|+|f(x, y, 0,0)|
$$

$F\left(L^{1}\left(I^{2}\right)\right) \subset L^{1}\left(I^{2}\right)$

Further, we have

$$
\begin{aligned}
& |F(w)(x, y)-F(v)(x, y)| \leqslant L_{1} \int_{D(h(x, y))}|w(t, s)-v(t, s)| d t d s \\
& \quad+L_{2}|w(H(x, y))-v(H(x, y))|, \quad w, v \in L^{1}\left(I^{2}\right), \quad(x, y) \in I^{2} .
\end{aligned}
$$

Thus

$$
\begin{aligned}
& \|F(w)-F(v)\|_{1} \\
& \leqslant L_{1} \int_{I^{2}}\left(\int_{D(h(x, y))}|w(t, s)-v(t, s)| d t d s\right) d x d y+L_{2} \int_{I^{2}}|w(H(x, y))-v(H(x, y))| d x d y \\
& \leqslant L_{1} a^{2}\|w-v\|_{1}+L_{2} \int_{I^{2}}|w(H(x, y))-v(H(x, y))|\left|H^{\prime}(x, y)\right| \frac{1}{\left|H^{\prime}(x, y)\right|} d x d y \\
& \leqslant\left(L_{1} a^{2}+L_{2} M\right)\|w-v\|_{1} .
\end{aligned}
$$

In view of Banach contraction principle the mapping $F$ has a unique fixed point. Hence the proof of Theorem 2 is completed.

\section{REMARKS}

It is clear that in the of proof Theorem 2 one can apply Proposition 1 (under additional assumption on the functions $h$ and $H$ ). Consider the following linear operator

$$
A(w)(x, y)=L_{1} \int_{D(h(x, y))} w(t, s) d t d s+L_{2} w(H(x, y))
$$


$w \in L^{1}\left(I^{2}\right),(x, y) \in I^{2}$

One can easy verify that if $h$ and $H$ satisfy $1^{0}$ and $2^{0}$ in addition then $\|A\|_{1} \leqslant$ $L_{1} a^{2}+L_{2} M,\left\|A^{2}\right\|_{1} \leqslant L_{1} a^{4} / 4+L_{1} L_{2} a^{2}(M+1)+L_{2}^{2} M$.

Further, it is well known that in an arbitrary Banach space

$$
r(A) \leqslant \sqrt[n]{\left\|A^{n}\right\|} \text { for every } n \in \mathbb{N}
$$

Hence, if for example, $M \geqslant 1$ then

$$
L_{1} a^{2}+L_{2} M \geqslant \sqrt{L_{1}^{2} \frac{a^{4}}{4}+L_{1} L_{2} a^{2}(M+1)+L_{2}^{2} M^{2}}
$$

and the assumption $r(A)<1$ is better than $L_{1} a^{2}+L_{2} M<1$. But we can not find an estimate of the spectral radius of the operator $A$ in terms of some constants and, therefore, we choose the Banach theorem to prove Theorem 2.

\section{REFERENCES}

[1] D.D. Bainov and D.P. Mishev, Oscillation theory for neutral differential equations with delay (Adam Hilger, Bristol, Philadelphia and New York, 1991).

[2] K. Deimling, 'Das Picard-Problem für $u_{x y}=f\left(x, y, u, u_{x}, u_{y}\right)$ unter CarathéodoryVoraussetzungen', Math. Z. 114 (1970), 303-312.

[3] K. Deimling, 'Das Goursat-Problem für $u_{x y}=f(x, y, u)$ ', Aequationnes Math. 6 (1971), 206-214.

[4] K.-H. Förster and B. Nagy, 'On the local spectral radius of a nonnegative element with respect to an irreducible operator', Acta Sci. Math. 55 (1991), 155-166.

[5] M.A. Krasnoselski, G.M. Vaimikko, P.P. Zabrieko, Ya.B. Rutitski and V.Ya Stetsenko, Approximate solutions of operator equations (Wolters-Noordhoff, Groningen, 1972).

[6] G.P. Tolstov, 'On the second mixed derivative', (in Russian), Mat. Sb. 24 (1949), 27-51.

[7] M. Zima, 'A certain fixed point theorem and its applications to integral-functional equations', Bull. Austral. Math. Soc. 46 (1992), 179-186.

[8] M. Zima, 'A theorem on the spectral radius of the sum of two operators and its application', Bull. Austral. Math. Soc. $\$ 8$ (1993), 427-434.

[9] M. Zima, 'On an integral equation with deviated arguments,', Demonstratio Math. 28 (1995), 967-973.

Faculty of Mathematics and Computer Science

Adam Michiewicz University

Poznań

Poland

e-mail: ddbb@math.amu.edu.pl
Department of Mathematics

Pedagogical University of Rzeszów

Rzeszów

Poland 